\title{
Research Paper: \\ Effect of Kangoo Jump on Posture Balance and Postural sway of Children with Autism
}

\author{
Paria Nekouei $^{1^{*}}$ (D) Vahid Zolaktaf ${ }^{1}$, Ebrahim Sadeghi-Demneh ${ }^{2}$ iD
}

1. Department of Exercise Rehabilitation, Faculty of Exercise Sciences, University of Isfahan, Isfahan, Iran.

2. Musculoskeletal Research Center, Isfahan University of Medical Sciences, Isfahan, Iran.

$\begin{gathered}\text { Use yur device to scan } \\ \text { and read the article online }\end{gathered}$
with Autism. Journal of Exercise Science and Medicine. 2020; 12(1):75-82. http://dx.doi.org/10.32598/JESM.12.1.8
woilhttp://dx.doi.org/10.32598/JESM.12.1.8

\section{(i) (5)}

Article info:

Received: 29 Mar 2019

Accepted: 06 May 2019

Available Online: 01 Jan 2020

Keywords:

Children, Autism Spectrum Disorder (ASD), Posture Balance, Postural Control, Postural Equilibrium

\section{A B S T RACT}

Introduction: Autism Spectrum Disorder (ASD) is among the most common childhood disorders. The current study aimed to investigate the effect of Kangoo Jump (KJ) exercises on dynamic balance and overall postural oscillations in children with ASD.

Materials and Methods: This was a quasi-experimental study. Among children with ASD in the Autism Association and Autism Schools in Tehran City, Iran, 20 subjects were selected using screening tests; after assessing the dynamic balance and general postural sway as a pretest, they were randomly divided into two groups of experimental and control. The experimental group performed KJ exercises for 8 weeks; eventually, all individuals were tested for dynamic balance and postural control as a posttest. The obtained data were analyzed using Analysis of Covariance (ANCOVA).

Results: This study suggested that performing KJ significantly improved the dynamic balance in children with an ASD disorder. There was a significant difference between the mean scores of the control and experimental groups in the posttest stage in dynamic equilibrium $(P<0.001)$; however, no difference was observed between the study groups in postural control.

Conclusion: Using KJ to improve dynamic balance in children with ASD is beneficial. Therefore these interventions can improve various aspects of development and balance in children with ASDs.

\footnotetext{
* Corresponding Author:
}

Paria Nekouei, PhD candidate.

Address: Department of Exercise Rehabilitation, Faculty of Exercise Sciences, University of Isfahan, Isfahan, Iran.

Tel: +98 (936) 7735290

E-mail: paria.nekouei@gmail.com 


\section{Introduction}

utism Spectrum Disorder (ASD) is among the neurodevelopmental disorders characterized by impaired social relationships, impaired communication, and repetitive behaviors [1]. The term "spectrum" reflects vast differences in the challenges and characteristics of an individual with autism [2]. The symptoms of ASD usually appear between the ages of 2 and 3 years. Besides, in some cases, it can be diagnosed as early as 18 months. The US Centers for Disease Control and Prevention estimates the prevalence of ASD as one in 68 children. This includes one in 42 boys and one in 189 girls. However, ASD is recognized as a social disorder [3].

Studies reported that children with ASD experience significant delays in developing motor skills [4]. Movement disorders in children with ASD affect their lives and cause problems with daily tasks, interaction with the physical environment, controlling fine movements, such as tying shoes and writing, increasing body composition, and elevated risk of diseases [5]. According to research, individuals with ASD manifest lower balance functions than normal children [6]. Maintaining a proper and natural balance of the body is critical in multiple daily activities and during exercise; it requires the interaction of sensory systems (visual, atrial, \& sensory body) and motor systems by the central nervous system. Applying appropriate intervention methods to improve it in individuals with disabilities is of significance [3].

Despite all deficiencies in children with ASD, relevant research suggested that appropriate interventions can positively affect these deficiencies. These interventions include physical activity. Schmitz et al. in a study of physical activity, concluded that such interventions could be useful for children with ASD [7].

One of the physical interventions that researchers have recently considered is the Kangoo Jump (KJ) exercise. $\mathrm{KJ}$ is a different practice whose challenge is balancing, and because it is fun and accompanied by music, it is a very attractive sport for children. Children are generally interested in jumping on springboards and trampolines. The KJ allows children to instill the same motor enthusiasm with the help of a special pair of shoes, without the need for complex and special tools. These shoes have numerous advantages. Among them, we can mention reducing the impact effect up to $87 \%$, the correction of the posture, and better coordination and apparent balance. Fluctuations in Kangoo shoes are per- manent jumps, and the body needs to cope with these fluctuations to maintain balance. This balance inactivity results from the body's balance system, sensory system, and visual system [8]. Additionally, accompanying music with $\mathrm{KJ}$ can accelerate balance in children with ASD.

Therefore, exploring these indicators by motor activities can be significant. There is insufficient research in this field and research that has examined physical activity in children with ASDs. Improvements in social relationships, communication, repetitive behaviors, and social interactions were less addressed in other developmental aspects, such as postural sway and balance [9].

The need to pay attention to the biopsychological health of children with special needs requires the plans and programs to improve their treatment and general rehabilitation. This is because they are always problematic for themselves, their parents, and school. Therefore, the necessary treatment strategies can help parents and educators in the rehabilitation of these children; one of which is to pay attention to the role of physical activity in the improvement and treatment of specific behavioral disorders in children. The mentioned materials are essential. There exists a lack of interventional research in this field in Iran. Furthermore, the prevalence of this disorder is on the rise. Thus, this study aimed to determine the effect of $\mathrm{KJ}$ on dynamic balance and general postural sway in children with ASDs.

\section{Materials and Methods}

This was a quasi-experimental study with a pretestposttest and a control group design. The present study's statistical population consisted of children (girls \& boys) with ASD in Tehran City, Iran. The research sample was selected by purposive sampling method based on the inclusion and exclusion criteria among children who were referred to the Autism Association of Tehran. The inclusion criteria of the research included the following: A subjects with ASD at the discretion of a psychologist based on the Gilliam 2-7 index, the age range of 7 to 12 years, with ASD, completing the consent form by parents, no medical prohibition to participate in the activity, and the absence of orthopedic and cardiorespiratory disorders. Among the exclusion criteria was not participating in the pretest and posttest stages and missing $>3$ training sessions.

Then, among all eligible children, 20 were randomly selected as the statistical sample. In this sampling method, each member of the statistical community has an equal chance of being selected. Prior to the interven- 
Table 1. Research plan

\begin{tabular}{|c|c|c|c|}
\hline Groups & Pretest & Intervention & Posttest \\
\hline Experimental & Balance measurement and postural sway & Kangoo Jump & Balance measurement and postural sway \\
\hline Control & Balance measurement and postural sway & $\begin{array}{l}\text { School routine } \\
\text { exercise }\end{array}$ & Balance measurement and postural sway \\
\hline
\end{tabular}

tion, all children were individually examined respecting dynamic balance by heel-to-toe test and postural sway by the force plate. The study participants were then randomly divided into the following groups: experimental (age: $9.80+1.31$ y) and control (age: $9.78+1.20$ $y)$. Before entering the research, in a session for parents and children in both groups, videos, movements were explained and illustrated by $\mathrm{KJ}$ instructors. The study sample received explanations on how to perform the movements used with KJ shoes during the course. Then, the parents and children who were still willing to participate in the research with full knowledge of the exercises and research method filled in the consent form and the research entry form and prepared to participate in the research (Table 1$)$.

In the present study, the training protocol was planned and implemented with international trainers and $\mathrm{KJ}$ instructors. The protocol used in the study was experimentally performed on a small group of children; it was prepared and processed for the study. The KJ Exercise Protocol includes KJ Exercise Protocol for eight weeks, 3 sessions per week, and 40 minutes per session. The type of exercise and the difficulty of each exercise changed every two weeks, after 6 sessions of each exercise. Each movement in the main part of the program consisted of 3 difficulty levels. Thus, at the end of the eighth week, 8 exercises were practiced by the research participants. A group of $\mathrm{KJ}$ trainers designed this program, and the previous source is unavailable. In the present program, the ratio of rest to exercise was considered three to one.

\section{The tools used in this study were as follows}

1. The Gilliam Autism Rating Scale - Second Edition (GARS-2) [3]: The Gars test is a checklist that helps diagnose individuals with an ASD. Gars test is suitable for individuals aged 3 to 33 years. The Gars test consists of 4 subscales, and each subscale contains 14 options. These subscales include stereotyped behaviors, communication, social interactions, and developmental disorders, i.e., completed based on direct observation and interviews with parents and teachers of children with ASD and used in educational and research programs.
In the present study, prepared scores and tests were taken in the archives of the Tehran Autism Association were used. The reliability of the Gars-2 internal stability is determined using Cronbach's alpha coefficient. Studies indicated an alpha of $84 \%$ for stereotyped behaviors, $86 \%$ for communication, $88 \%$ for social interaction, and 94\% for ASDs [10]. In Iranian society, based on the study of Ahmadi et al., Cronbach's alpha coefficient in stereotyped behavior was measured to be $74 \%$, communication as $92 \%$, social interactions as $73 \%$, and growth issues as $80 \%$. Cronbach's alpha coefficient of the test was computed to be $89 \%$. This coefficient indicates the high reliability of the Gars scale, which can be used for diagnostic and therapeutic purposes [11].

2. Force plate: Kistler force plates (Kistler, Switzerland; $500 \times 600 \mathrm{~mm}$, model SA 960) was used to test the postural sway in the AP and ML plates. For this purpose, the force plate device was first calibrated and tested at a frequency of $120 \mathrm{~Hz}$. Additionally, before testing, the device was filtered with a frequency of $10 \mathrm{~Hz}$ to pick up noise. During the standing tests, the position of the foot was determined and used for all subsequent tests. To measure postural stability, the study subjects were requested to stand barefoot on the force plate for 60 seconds. The children were instructed to stand comfortably with their hands on the body and look at the lab wall quietly. The test was repeated twice; between each repetition, the examined subjects were rested on a chair already installed in the test site for 30 seconds. Then, because the centre of pressure (CoP) moves a lot in the first 15 seconds, also to eliminate the effect of possible fatigue created in the last 15 seconds, the middle 30 seconds were used as the test result. Therefore, 1800 frames $(15 \mathrm{~s} \times 120 \mathrm{~Hz}=1800)$ to 5400 frames $(45 \mathrm{~s} \times 120 \mathrm{~Hz}=5400)$ were used for COP analysis. Accordingly, 3600 frames $(30 \mathrm{~s} \times 120 \mathrm{~Hz}=3600)$ in each iteration were examined. In the analysis, the parameters of COP motion length, COP oscillation rate, total COP motion length, and total COP oscillation rate were used in AP and ML plates [12].

3. Heel-to-toe walking test (tandem gait): To assess dynamic balance, a heel-to-toe walk test was used. In this test, the ability of individuals is measured in a di- 
rect path from heel to toe. Individuals were requested to walk from heel to toe on a specific path 15 feet long. If the explored individuals deviated or deviated from the path before completing the 15 steps, the test would be stopped and the number of steps recorded as their score. This test was performed twice, and the best score was considered as a record [13]. The reliability of this test was calculated by Salar et al. as 0.91\% [14].

The obtained data were analyzed using descriptive statistics (Mean $\pm S D$ ) and inferential statistics Analysis of Covariance (ANCOVA) in SPSS at a significance level of 0.05.

\section{Results}

First, in the descriptive statistics section, demographic characteristics were examined. Table 2 presents the values related to the descriptive indicators of the participants, such as the Mean \pm SD age, height, and weight of all participants in the research groups.

According to Table 2, the mean age of the experimental and control groups was 9.78 and 7.10 years, respectively. The mean height in the experimental group was $129.22 \mathrm{~cm}$, and in the control group, it was $131.60 \mathrm{~cm}$; the mean weight of the experimental group was 27.11 $\mathrm{kg}$, and in the control group it equaled $29.60 \mathrm{~kg}$. According to the Independent Samples t-test (Table 3), there was no significant difference between the study groups in the mentioned variables.

To determine the intergroup differences in the dynamic equilibrium test and the general postural sway, ANCOVA was used; the results of which are indicated in Tables 4 and 5.

As per Tables 4, the ANCOVA results revealed a statistically significant difference between the study groups in the posttest step after removing the pretest effect $(\mathrm{P}<0.001)$. Therefore, the intervention of $\mathrm{KJ}$ exercises has affected the dynamic balance.

As per Table 5, the ANCOVA results indicated no significant difference between the study groups after removing the pretest effect. Therefore, the intervention of KJ exercises did not affect the overall sway of posture.

\section{Discussion}

The present study investigated the effects of $\mathrm{KJ}$ exercises on dynamic balance and postural sway in children with ASD. The present study results suggested that following KJ exercises, dynamic balance improved in children with ASD. Thus, exercise and physical activity provide a great impact on the motor skills of these children and can improve motor performance in these

Table 2. Pretest and posttest data of the research variables and the characteristics of participants

\begin{tabular}{|c|c|c|c|c|}
\hline \multirow{2}{*}{ Variables } & \multicolumn{2}{|c|}{ Mean士SD } & \multirow{2}{*}{$\mathbf{t}$} & \multirow{2}{*}{$\mathbf{P}$} \\
\hline & Experimental Group ( $n=9)$ & Control Group $(n=10)$ & & \\
\hline Age (y) & $9.80 \pm 1.31$ & $9.78 \pm 1.20$ & 0.136 & 0.900 \\
\hline Height (cm) & $131.60 \pm 3.77$ & $129.22 \pm 4.14$ & 0.940 & 0.361 \\
\hline Weight (Kg) & $29.60 \pm 4.55$ & $27.11 \pm 5.44$ & 0.311 & 0.763 \\
\hline
\end{tabular}

Exercise Science and Medicine

Table 3. Descriptive indices of dynamic balance variables and overall postural sway

\begin{tabular}{cccc}
\hline \multirow{2}{*}{ Variables } & Groups & \multicolumn{2}{c}{ Mean \pm SD } \\
\cline { 3 - 4 } & & Pretest & Posttest \\
\hline Dynamic balance/Heel to toe & Experimental & $8.11 \pm 1.36$ & $9.88 \pm 1.26$ \\
& Control & $7.70 \pm 1.15$ & $7.60 \pm 1.42$ \\
Postural Sway/Force plate & Experimental & $4.79 \pm 1.11$ & $4.59 \pm 1.09$ \\
& Control & $4.49 \pm 1.17$ & $4.87 \pm 1.27$ \\
\hline
\end{tabular}


Table 4. Results of ANCOVA to compare the post-test of the mean scores of the dynamic balance variables

\begin{tabular}{cccc} 
Source & F & P & Partial Eta-Squared \\
\hline Dynamic balance_pre & 17.183 & 0.001 & 0.518 \\
Group & 18.941 & 0.001 & 0.542 \\
\hline & & & Journal of \\
& & Exercise Science and Medicin€
\end{tabular}

Table 5. ANCOVA results compare the posttest of the mean values of postural sway variables

\begin{tabular}{|c|c|c|c|}
\hline Sources & $\mathbf{F}$ & $\mathbf{P}$ & Partial Eta-Squared \\
\hline Postural sway_pre & 38.623 & 0.001 & 0.707 \\
\hline Group & 0.048 & 0.829 & 0.003 \\
\hline
\end{tabular}

children. Children with ASDs are prone to impaired balance, postural stability, and postural abnormalities. The symptoms of this disorder appear in the first 3 years of life. As mentioned, ASD is also a developmental disorder that can potentially affect cognitive-motor abilities as well as motor skills [15].

According to research conducted over the last 2 decades, the percentage increase in ASD was associated with significant growth. However, these children can be helped with early diagnosis, greater awareness, newer treatment options, and special educational methods. Pharmacological interventions for individuals with ASD are mainly used to reduce autism-related symptoms, such as inattention, impulsivity, hyperactivity, anxiety, sleep disorders, irritability, and self-harm [15]. There is currently no approved drug to treat the symptoms of ASD. Numerous research groups worldwide are focused on finding the best safe medicine for individuals with ASD. According to past research, children with ASD are increasingly sedentary [16]; therefore, recognizing effective interventions can help to improve the body's metabolism, cardiovascular, and cognitive health [17].

Numerous daily motor activities are facilitated by the repetition and practice of basic skills in childhood; thus, it is necessary for the child to freely explore the environment and interact with others [18]. The symptoms of children with ASD include less interaction with their peers and no participation in group games, compared to the typically-developing children. Therefore, because children with autism are less present in the community and have fewer motor experiences, it is obvious that they have poor motor skills [19].
$\mathrm{KJ}$ is a different sport whose challenge is balancing. Moreover, because it is fun and accompanied by music, it is a very attractive sport for children. Children are generally interested in jumping on springboards and trampolines. The $\mathrm{KJ}$ allows children to instill the same motor enthusiasm with the help of a special pair of shoes, without the need for complex and special tools. These shoes have multiple advantages. Among them, we can mention reducing the impact effect up to $87 \%$, the correction of the posture, and better coordination and apparent balance. Fluctuations in Kangoo shoes are permanent jumps, and the body must cope with these fluctuations to maintain balance. This balance inactivity results from the body's balance system, sensory system, and visual system [8]. Considering that balance requires more attention than other motor skills, it requires further practice to progress and improve. The impact of the $\mathrm{KJ}$ protocol with music, i.e., tailored to the needs of the subjects, could be among the main reasons why children with ASD develop balance skills in this study, according to Kim et al. [20] is also presented.

One of the possible mechanisms for the success of the present study's training protocol in improving children's balance with ASDs was the KJ training protocol on movements that required balance. Other reasons for increasing the balance of children with ASD in this study include improving physical fitness factors, such as muscle strength, balance, coordination, and flexibility due to these exercises. Moreover, in most of these children, walking on the toes causes muscle shortening. Muscle stretching and relieving shortness will improve the child's condition is performed with the right exercises and at the right time. Practicing KJ with music may have helped overcome this defect and increase balance. This finding was consistent with those of Dorunjic [21], 
indicating that physical activity positively affects motor function in children with ASDs.

Mateos et al. [22], in a study as a combination of music and exercise on individuals with ASD, found that the association of music with motor activities is a kind of motor training and functional balance with more neuromuscular function, i.e., enjoyable for individuals. At the same time structure of music, including rhythmic sounds in a stable pattern, facilitates body movements, improves motor skills, increases eye-hand coordination, strengthens and controls movements, as well as improves joint range of motion. As a result, it improves motor skills, like balance in children with ASD [23]. The use of music, as mentioned earlier, during physical activity in most cases improves and increases participation in the learning and social interactions of children with ASD; thus, it improves their functional independence helps, which these factors can also play a significant role in improving the motor skills of these children [24]. Exercise with music is associated with improved balance and gait in individuals with ASD.

Neurophysiological studies revealed that defects in cortical and subcortical areas such as the motor cortex, the complementary motor cortex, basal ganglia, and cerebellum can cause motor design defects, sensory-motor integration, and movement performance. Qiu et al. [24] concluded that motor, social, and communication deficits are associated with the basal ganglia's abnormal shape. Defects in the atrial and visual systems can also limit the connection between the visual and atrial senses' afferent systems; ultimately, the imbalance in posture [24]. KJ, as an activity that requires learning skills, motor control, and social engagement, may be related to cerebellar function and cortical and subcortical areas. Numerous studies revealed improved motor skills after long-term and regular intervention of various physical activities in children with ASD. In general, motor activities can help improve these children's motor skills. This result was in line with those of the present study; as it was observed, KJ exercises could improve balance function in children with ASD.

In addition to the dynamic balance examined in the present study, postural sway was also examined. The obtained results suggested no significant difference between the control and experimental groups. Thus, $\mathrm{KJ}$ exercises did not significantly affect postural sway in individuals with an ASD. Since KJ is a new sport both in Iran and worldwide, the sport is still not part of public sports, and there has not been many studies on that. Due to the limited availability of studies in this area, it is impossible to speak confidently in this regard. As a result, further research is necessary to conclude a definitive conclusion in this area.

\section{Conclusion}

The present study results indicated that following a course of KJ, dynamic balance improves in children with ASD. Thus, exercise and physical activity provide a great impact on the motor skills of these children and can lead to improved motor performance in them. Therefore, such interventions can improve various aspects of development and balance in children with ASD.

Despite the significant positive results, some limitations of the study should be considered when generalizing the present study results. Dynamic balance improvement was inferred using a comparison of posttest session measurement with a pretest. Therefore, no judgment can be made about the continuity of the results over time. These effects may diminish over time and may not be permanent.

\section{Ethical Considerations}

\section{Compliance with ethical guidelines}

All procedures performed in studies involving human participants were in accordance with the ethical standards of the institutional and/or national research committee and with the 1964 Helsinki declaration and its later amendments or comparable ethical standards.

\section{Funding}

This work was 'extracted from the doctorate dessertation, and have no funding support.

\section{Authors' contributions}

Conceptualization, Methodology, Investigation, Writing Original Draft: Paria Nekouei; Writing - Review \& Editing, All author; Supervision: Vahid Zolaktaf, Ebrahin Sadeghi Demneh.

\section{Conflict of interest}

The authors declared no conflict of interest.

\section{Acknowledgments}

We are grateful for the support and assistance of all the participating children and their families who assisted us in conducting this study. 


\section{References}

[1] Jia F, Wang B, Shan L, Xu Z, Staal WG, Du L. Core symptoms of autism improved after vitamin D supplementation. Pediatrics. 2015; 135(1):e196-8. [DOI:10.1542/peds.2014-2121] [PMID]

[2] Mottron L, Bzdok D. Autism spectrum heterogeneity: Fact or artifact? Molecular Psychiatry. 2020; 25(12):3178-85. [DOI:10.1038/ s41380-020-0748-y] [PMID] [PMCID]

[3] American Psychiatric Association. Diagnostic and statistical manua of mental disorders. Washington, D.C.: American Psychiatric Publishing; 2013. https://books.google.com/books?id=-JivBAAAQBAJ\&dq

[4] Ketcheson L, Hauck J, Ulrich D. The effects of an early motor skill intervention on motor skills, levels of physical activity, and socialization in young children with autism spectrum disorder: A pilot study. Autism. 2107; 21(4):481-92. [DOI:10.1177/1362361316650611] [PMID]

[5] Bremer E, Crozier M, Lloyd M. A systematic review of the behavioural outcomes following exercise interventions for children and youth with autism spectrum disorder. Autism. 2016; 20(8):899-915. [DOI:10.1177/1362361315616002] [PMID]

[6] Liu T, Breslin CM. Fine and gross motor performance of the MABC-2 by children with autism spectrum disorder and typically developing children. Research in Autism Spectrum Disorders. 2013; 7(10):1244-9. [DOI:10.1016/j.rasd.2013.07.002]

[7] Schmitz Olin S, Mcfadden BA, Golem DL, Pellegrino JK, Walker AJ, Sanders DJ, et al. The effects of exercise dose on stereotypical behavior in children with autism. Medicine and Science in Sports and Exercise. 2017; 49(5):983-90. [DOI:10.1249/MSS.0000000000001197] [PMID]

[8] de Oliveira RF, de Oliveira PD, Fernandes Szezerbaty SK, de Oliveira LC, de Almeida JS, de Oliveira Gil AW, et al. Effect of running exercise with and without the use of equipment Kangoo Jumps, in postural control: A case study. Manual Therapy, Posturology \& Rehabilitation Journal. 2014; 131-5. [DOI:10.17784/mtprehabjournal.2014.12.179]

[9] Movahedi A, Bahrami F, Marandi SM, Abedi A. Improvement in social dysfunction of children with autism spectrum disorder following long term Kata techniques training. Research in Autism Spectrum Disorders. 7(9):1054-61. [DOI:10.1016/j.rasd.2013.04.012]

[10] Montgomery JM, Newton B, Smith C. Test Review: Gilliam, J. (2006). GARS-2: Gilliam Autism Rating Scale-Second Edition. Austin, TX: PRO-ED. Journal of Psychoeducational Assessment. 2008; 26(4):395-401. [DOI:10.1177/0734282908317116]

[11] Ahmadi SJ, Safari T, Hemmatian M, Khalili Z. [The psychometric properties of Gilliam Autism Rating Scale (GARS) (Persian)]. Research in Cognitive and Behavioral Sciences. 2011; 1(1):87-104. https://cbs.ui.ac.ir/article_17282_en.html

[12] Pan CY, Tsai $\mathrm{CL}$, Chu CH. Fundamental movement skills in children diagnosed with autism spectrum disorders and attention deficit hyperactivity disorder. Journal of Autism and Developmental Disorders. 2009; 39(12):1694-705. [DOI:10.1007/s10803-009-0813-5] [PMID]

[13] Cannell JJ. Autism, will vitamin D treat core symptoms? Medical Hypotheses. 2013; 81(2):195-8. [DOI:10.1016/j.mehy.2013.05.004] [PMID]

[14] Salar S, Daneshmandi H, Panaghi L, Karimizadeh Ardekani M, Nazari Sharif $\mathrm{H}$. The investigation of the relationship between core endurance with static and dynamic balance in children with autism spectrum disorder. International Journal of Sport Studies. 2015; 5(1):48-56. https://www.sid.ir/en/Journal/ViewPaper.aspx?ID=554100
[15] Healy S, Haegele JA, Grenier M, Garcia JM. Physical activity, screen-time behavior, and obesity among 13-year olds in ireland with and without autism spectrum disorder. Journal of Autism and Developmental Disorders. 2017; 47(1):49-57. [DOI:10.1007/ s10803-016-2920-4] [PMID]

[16] Happé F, Booth R, Charlton R, Hughes C. Executive function deficits in autism spectrum disorders and attention-deficit/hyperactivity disorder: Examining profiles across domains and ages. Brain and Cognition. 2006; 61(1):25-39. [DOI:10.1016/j.bandc.2006.03.004] [PMID]

[17] Taheri M. [Comparison of reaction time and balance in autism and normal children (Persian)]. Rooyesh-e- Ravanshenasi Journal. 2016; 4(4):49-60. [DOI:20.1001.1.2383353.1394.4.4.6.0]

[18] Mosconi MW, Mohanty S, Greene RK, Cook EH, Vaillancourt DE, Sweeney JA. Feedforward and feedback motor control abnormalities implicate cerebellar dysfunctions in autism spectrum disorder. The Journal of Neuroscience. 2015; 35(5):2015-25. [DOI:10.1523/ JNEUROSCI.2731-14.2015] [PMID] [PMCID]

[19] Brito ACM. Treinamento internacional kangoo jumps fitness instructor. 2010. https://scholar.google.com/scholar?hl=en\&as_sdt= $0 \% 2 C 5 \& q=$ Treinamento+Internacional+Kangoo+Jumps+Fitness+In structor\&btnG=

[20] Kim Y, Todd T, Fujii T, Lim JC, Vrongistinos K, Jung T. Effects of Taekwondo intervention on balance in children with autism spectrum disorder. Journal of Exercise Rehabilitation. 2016; 12(4):314-9. [DOI:10.12965/jer.1632634.317] [PMID] [PMCID]

[21] Duronjić $M$, Válková $H$. The influence of early intervention movement programs on motor skills development in preschoolers with autism spectrum disorder (case studies). Acta Universitatis Palackianae Olomucensis Gymnica. 2010; 40(2):37-45. https://www.gymnica.upol.cz/pdfs/gym/2010/02/04.pdf

[22] Mateos-Moreno D, Atencia-Doña L. Effect of a combined dance/ movement and music therapy on young adults diagnosed with severe autism. The Arts in Psychotherapy. 2013; 40(5):465-72. [DOI:10.1016/j.aip.2013.09.004]

[23] Fong CE, Jelas ZM. Music education for children with autism in Malaysia. Procedia - Social and Behavioral Sciences. 2010; 9:70-5. [DOI:10.1016/j.sbspro.2010.12.117]

[24] Qiu A, Adler M, Crocetti D, Miller MI, Mostofsky SH. Basal ganglia shapes predict social, communication, and motor dysfunctions in boys with autism spectrum disorder. Journal of the American Academy of Child and Adolescent Psychiatry. 2010; 49(6):539-51. [DOI:10.1016/j.jaac.2010.02.012] [PMID] 
This Page Intentionally Left Blank 